\title{
A TRACE FORMULA FOR TWO UNITARY OPERATORS WITH RANK ONE COMMUTATOR
}

\author{
KHRISTO N. BOYADZHIEV
}

(Communicated by Paul S. Muhly)

\begin{abstract}
We give a short and independent proof of the Carey-Pincus trace formula for pairs of unitary operators $U, V$ with $\operatorname{Rank}[U, V]=1$.
\end{abstract}

Theorem. Let $U, V$ be two unitary operators with a one-dimensional commutator on a complex Hilbert space $H$. Then there exists a real measurable function $f(t, s)$ defined for $t, s \in[0,2 \pi]$ with values in the interval $[0,1]$ such that the formula

$$
\operatorname{tr}([p(U, V), q(U, V)])=\frac{1}{2 \pi i} \int_{0}^{2 \pi} \int_{0}^{2 \pi} \frac{D(p, q)}{D(t, s)}\left(e^{i t}, e^{i s}\right) f(t, s) d t d s
$$

holds for any two polynomials $p, q$ of the form

$$
p\left(e^{i t}, e^{i s}\right)=\sum c_{n, n} e^{i n t} e^{i m s} \text { with } p(U, V)=\sum c_{n, m} U^{n} V^{m},
$$

and $D(p, q) / D(t, s)$ is the Jacobian of $p, q$ with respect to $t, s$. The function $f$ also has the property: $f(t, s)=0$ if $e^{i t} \notin \sigma(U)$ or $e^{i s} \notin \sigma(V)$.

Comments. Trace formulas for commutators of selfadjoint operators $A, B$ with $[A, B] \in L^{1}(H)$, the trace class, have been obtained by Carey and Pincus [5, 15] and Helton and Howe [12] (see also [2, 4, 7, 14, 19]). For pairs of unitary operators $U, V$ with a trace class commutator, formula (1), with $f$ integrable, was proved in the general setting of the type II $_{\infty}$ case by Carey and Pincus [6]. Their proof is based on the introduction of a determining function for the pair $U, V$ and applies a modification of the method presented in [5].

We introduce here a new approach based on the function

$$
\begin{array}{r}
k(\lambda, \mu)=\frac{1}{2 \pi i} \log \operatorname{det}\left(U_{\lambda} V_{\mu} U_{\lambda}^{-1} V_{\mu}^{-1}\right)=\frac{1}{2 \pi i} \operatorname{tr} \log \left(U_{\lambda} V_{\mu} U_{\lambda}^{-1} V_{\mu}^{-1}\right), \\
|\lambda|<1,|\mu|<1,
\end{array}
$$

Received by the editors March 12, 1990.

1980 Mathematics Subject Classification (1985 Revision). Primary 45B15. 
where the unitary operators $U_{\lambda}, V_{\mu}$ are defined by

$$
\begin{aligned}
& U_{\lambda}=(U-\bar{\lambda})(1-\lambda U)^{-1}=\frac{1-|\lambda|^{2}}{\lambda}(1-\lambda U)^{-1}-\frac{1}{\lambda} \\
& V_{\mu}=(V-\bar{\mu})(1-\mu V)^{-1}=\frac{1-|\mu|^{2}}{\mu}(1-\mu V)^{-1}-\frac{1}{\mu} .
\end{aligned}
$$

This is a real-valued function defined in the polydisk $D=\{\lambda, \mu \in C:|\lambda|<1$, $|\mu|<1\}$ and direct differentiation by the chain rule shows that $k(\lambda, \mu)$ is harmonic in $\lambda$ and $\mu$ separately. Further properties of $k$ can be obtained by relating it to the Krein spectral shift function, as this is done in [3]. Thus one finds $|k(\lambda, \mu)|<n$, when Range $[U, V]=n<\infty$ and $k \in H^{1}(D)$ when $[U, V] \in L^{1}(H)$. In these cases $k$ admits a representation by a double Poisson integral (see (7) below) and by differentiating this representation we come to the desired trace formula. At that, the principal function $f(t, s)$ in (1) is determined by the boundary values of $k$.

In order to illustrate this method we consider the case when $\operatorname{Range}[U, V]=1$ which is very simple and can be treated directly. Although simple, this case is the most important one, (see [8, 9, 14, 16, 17, 20, 21]), has a rich theory, and provides a good insight in the nature of $f(t, s)$.

Proof of the theorem. It is easy to check that

$\left[U_{\lambda}, V_{\mu}\right]=\left(1-|\lambda|^{2}\right)\left(1-|\mu|^{2}\right)(1-\lambda U)^{-1}(1-\mu V)^{-1}[U, V](1-\mu V)^{-1}(1-\lambda U)^{-1}$.

We want to show that the unitary operator

$$
W_{\lambda \mu}=U_{\lambda} V_{\mu} U_{\lambda}^{-1} V_{\mu}^{-1}=1+\left[U_{\lambda}, V_{\mu}\right] U_{\lambda}^{-1} V_{\mu}^{-1}
$$

has, for all $\lambda, \mu$, just two different eigenvalues; namely, one is 1 and the other we denote by $\alpha(\lambda, \mu)$ (cf. [6, Example (3.12), p. 76]. This is obvious: the operator $\left[U_{\lambda}, V_{\mu}\right] U_{\lambda}^{-1} V_{\mu}^{-1}$ is one-dimensional and has at most one eigenvalue $\alpha(\lambda, \mu)-1$ different from 0 and no continuous spectrum. If for some $\lambda, \mu, W_{\lambda \mu}$ has only the eigenvalue 1 , that is $\alpha(\lambda, \mu)=1$, then $W_{\lambda \mu}$ will be the identity operator and $\left[U_{\lambda}, V_{\mu}\right]=0$, which is impossible, as $[U, V] \neq 0$. Therefore $\alpha(\lambda, \mu) \neq 1$ for all $(\lambda, \mu) \in D$. We see that when $\lambda, \mu$ vary in the open unit disk, $\alpha(\lambda, \mu)$ varies continuously on the unit circle never reaching the point 1 and hence never passing through it. We can write

$$
\alpha(\lambda, \mu)=\operatorname{det}\left(W_{\lambda \mu}\right)=\exp (2 \pi i k(\lambda, \mu)), \quad|\lambda|,|\mu|<1,
$$

where

$$
k(\lambda, \mu)=\frac{1}{2 \pi i} \log \operatorname{det}\left(W_{\lambda \mu}\right)=\frac{1}{2 \pi i} \operatorname{tr} \log \left(W_{\lambda \mu}\right)
$$

is a continuous function taking values in the interval $(0,1)$. For $\log (\cdot)$ we take the branch holomorphic on $\mathbf{C} \backslash R^{+}$. (See also Note (a) at the end.) Differentiating $\operatorname{tr} \log \left(U_{\lambda} V_{\mu} U_{\lambda}^{-1} V_{\mu}^{-1}\right)$ by the chain rule (see [10, 1.6.11, Lemma 3] 
or $\left.\left[11, \mathrm{IV} .1 .9^{\circ}\right]\right)$ we find that $k(\lambda, \mu)$ is harmonic in $\lambda$ and $\mu$ separately (the differentiation of $k$ is given in Note (c) at the end), that is

$$
\frac{\partial^{2}}{\partial \lambda \partial \bar{\lambda}} k(\lambda, \mu)=\frac{\partial^{2}}{\partial \mu \partial \bar{\mu}} k(\lambda, \mu)=0 \quad \text { in } D \text {. }
$$

Being bounded and separately harmonic, the function $k(\lambda, \mu)$ has nontangential boundary values on the unit circles $|\lambda|=1,|\mu|=1$. In particular

$$
k\left(e^{i t}, e^{i s}\right)=\lim _{r \rightarrow 1-\rho \rightarrow 1-} \lim k\left(r e^{i t}, \rho e^{i s}\right) \text { for a.e. } t, s \in[0,2 \pi],
$$

and the limits can be interchanged. Also, $k$ admits the representation by a double Poisson integral

$$
k(\lambda, \mu)=\frac{1}{4 \pi^{2}} \int_{0}^{2 \pi} \int_{0}^{2 \pi} P_{r}(\theta-t) P_{\rho}(\varphi-s) k\left(e^{i t}, e^{i s}\right) d t d s
$$

where $\lambda=r e^{i \theta}, \mu=\rho e^{i \varphi}$, and $P_{r}(\theta-t)=\frac{1-r^{2}}{1+r^{2}-2 r \cos (\theta-t)}=\operatorname{Re}\left\{\frac{e^{i t}+\lambda}{e^{i t}-\lambda}\right\}$

is the Poisson kernel for the unit disk (see $[2,13,18]$ ).

This formula is interesting by itself. Combined with (4) it provides a "real part" version for unitary operators of the exponential formula considered, for instance, in [8] and [17].

We proceed further by applying the differential operator $\partial^{2} / \partial \lambda \partial \mu$ to both sides of (7) written in the form

$$
\operatorname{tr} \log \left(U_{\lambda} V_{\mu} U_{\lambda}^{-1} V_{\mu}^{-1}\right)=\frac{i}{2 \pi} \iint \operatorname{Re}\left\{\frac{e^{i t}+\lambda}{e^{i t}-\lambda}\right\} \operatorname{Re}\left\{\frac{e^{i s}+\mu}{e^{i s}-\mu}\right\} k\left(e^{i t}, e^{i s}\right) d t d s
$$

(integration everywhere will be for $t, s \in[0,2 \pi]$ as in (7)). This gives

$$
\operatorname{tr}\left[U(1-\lambda U)^{-1}, V(1-\mu V)^{-1}\right]=\frac{i}{2 \pi} \iint \frac{e^{i t}}{\left(e^{i t}-\lambda\right)^{2}} \frac{e^{i s}}{\left(e^{i s}-\mu\right)^{2}} k\left(e^{i t}, e^{i s}\right) d t d s .
$$

Expanding both sides in powers of $\lambda$ and $\mu$ and comparing coefficients, one finds that for all nonnegative integers $n, m$ :

$$
\begin{aligned}
\operatorname{tr}\left[U^{n}, V^{m}\right] & =\frac{i}{2 \pi} \iint n m e^{-i n t} e^{-i m s} k\left(e^{i t}, e^{i s}\right) d t d s \\
& =\frac{i}{2 \pi} \iint \frac{\partial}{\partial t}\left(e^{i n t}\right) \frac{\partial}{\partial s}\left(e^{i m s}\right) k\left(e^{-i t}, e^{-i s}\right) d t d s
\end{aligned}
$$

(after the substitution $t \rightarrow 2 \pi-t, s \rightarrow 2 \pi-s$ ).

Applying successively to (8) the operators $\partial^{2} / \partial \bar{\lambda} \partial \mu, \partial^{2} / \partial \lambda \partial \bar{\mu}, \partial^{2} / \partial \bar{\lambda} \partial \bar{\mu}$, and arguing in the same way, we find that (10) holds for all integers $n, m \in Z$.

Finally, a standard application of Wallach's lemma implies the trace formula (1) with $f(t, s)=k\left(e^{-i t}, e^{-i s}\right)$ (see $\left.[5,7,12,14,19]\right)$.

If $e^{i t} \notin \sigma(U)$, then the operator $\left(1-e^{-i t} U\right)^{-1}$ is bounded and when $\lambda \rightarrow$ $e^{-i t}$ and $\mu$ stays fixed, (2) implies $\left[U_{\lambda}, V_{\mu}\right] \rightarrow 0$ and hence $k(\lambda, \mu) \rightarrow 0$. The 
same is true when we interchange $U$ and $V, e^{i t}$ and $e^{i s}, \lambda$ and $\mu$. Therefore $f(t, s)=k\left(e^{-i t}, e^{-i s}\right)=0$ when $e^{i t} \notin \sigma(U)$ or $e^{i s} \notin \sigma(V)$ according to (6). The proof is completed.

Some notes. (a) The operator $W_{\lambda \mu}=U_{\lambda} V_{\mu} U_{\lambda}^{-1} V_{\mu}^{-1},|\lambda|,|\mu|<1$, being unitary, has the representation

$$
\begin{aligned}
& W_{\lambda \mu}=\int_{0}^{2 \pi} e^{i \theta} d E_{\lambda \mu}(\theta)=\exp \left(i A_{\lambda \mu}\right) \\
& \text { where } A_{\lambda \mu}=\int_{0}^{2 \pi} \theta d E_{\lambda \mu}(\theta)=\frac{1}{i} \log \left(W_{\lambda \mu}\right)
\end{aligned}
$$

In case $[U, V]$ is one-dimensional, $\alpha(\lambda, \mu)$ is the only eigenvalue of $W_{\lambda \mu}$ different from 1 and $2 \pi k(\lambda, \mu)$ is the only eigenvalue of $A_{\lambda \mu}$ different from 0 (the spectral function $E_{\lambda \mu}(\theta)$ has exactly one jump for $\left.0<\theta<2 \pi\right)$. When $[U, V]$ is $n$-dimensional, $n>1, E_{\lambda \mu}$ has (at most) $n$ jumps in $(0,2 \pi)$ and hence $A_{\lambda \mu}$ has (at most) $n$ nonzero eigenvalues $2 \pi k_{j}(\lambda, \mu), j=1,2, \ldots, n$, $0<k_{j}<1$. In this case

$$
k(\lambda, \mu)=\frac{1}{2 \pi} \operatorname{tr}\left(A_{\lambda \mu}\right)=\sum k_{j}(\lambda, \mu) \in(0, n)
$$

and we get (1) with $0 \leqq f \leqq n$. Also, for $\lambda=\mu=0$ we find from (8)

$$
\frac{2 \pi}{i} \operatorname{tr} \log \left(U V U^{*} V^{*}\right)=\iint f(t, s) d t d s .
$$

(b) We give $\alpha(\lambda, \mu)$ in explicit form. As the normal operator [ $U, V] U^{-1} V^{-1}$ $=U V U^{-1} V^{-1}-1$ is one-dimensional, there exist a nonzero vector $\psi \in H$ and a nonzero complex number $a$, such that $[U, V] U^{-1} V^{-1} x=a(x, \psi) \psi$ and $V U[U, V]^{*} x=\left([U, V] U^{-1} V^{-1}\right)^{*} x=\bar{a}(x, \psi) \psi$ for all $x \in H$ (see $[1, \S 6]$.

Define the vector function of norm one

$$
\psi(\lambda, \mu)=(1-\lambda U)^{-1}(1-\mu V)^{-1} \psi /\left\|(1-\lambda U)^{-1}(1-\mu V)^{-1} \psi\right\|, \quad|\lambda|,|\mu|<1 .
$$

In view of $(2), \psi(\lambda, \mu)$ is a normalized eigenvector of the one-dimensional operator $\left[U_{\lambda}, V_{\mu}\right] U_{\lambda}^{-1} V_{\mu}^{-1}=U_{\lambda} V_{\mu} U_{\lambda}^{-1} V_{\mu}^{-1}-1=W_{\lambda \mu}-1$, and hence of $W_{\lambda \mu}$. For the corresponding eigenvalue $\alpha(\lambda, \mu)$ we have

$$
\alpha(\lambda, \mu)=\left\langle W_{\lambda \mu} \psi(\lambda, \mu), \psi(\lambda, \mu)\right\rangle,
$$

and one can check directly that $\operatorname{Re} \alpha(\lambda, \mu)<1$ in $D$. 
(c) Differentiation of $k(\lambda, \mu)$. It is sufficient to find $\partial k(\lambda, \mu) / \partial \lambda$. Because $\partial U_{\lambda} / \partial \lambda=(U-\bar{\lambda})(1-\lambda U)^{-2} U=U(1-\lambda U)^{-1} U_{\lambda}$ and because $\partial U_{\lambda}^{-1} / \partial \lambda=$ $-U(U-\bar{\lambda})^{-1}=-U(1-\lambda U)^{-1} U_{\lambda}^{-1}$, we find

$$
\begin{aligned}
& \frac{\partial}{\partial \lambda} \\
& 2 \pi i k(\lambda, \mu)=\frac{\partial}{\partial \lambda} \operatorname{tr} \log \left(W_{\lambda \mu}\right)=\operatorname{tr}\left[\left(\frac{\partial}{\partial \lambda} W_{\lambda \mu}\right) W_{\lambda \mu}^{-1}\right] \\
& \left.\quad=\operatorname{tr}\left\{U(1-\lambda U)^{-1} U_{\lambda} V_{\mu} U_{\lambda}^{-1} V_{\mu}^{-1}-U_{\lambda} V_{\mu} U(1-\lambda U)^{-1} U_{\lambda}^{-1} V_{\mu}^{-1}\right) V_{\mu} U_{\lambda} V_{\mu}^{-1} U_{\lambda}^{-1}\right\} \\
& \quad=\operatorname{tr}\left(U(1-\lambda U)^{-1}-U_{\lambda} V_{\mu} U(1-\lambda U)^{-1} V_{\mu}^{-1} U_{\lambda}^{-1}\right) \\
& \quad=\operatorname{tr}\left\{U_{\lambda}\left(U_{\lambda}^{-1} U(1-\lambda U)^{-1} U_{\lambda}-V_{\mu} U(1-\lambda U)^{-1} V_{\mu}^{-1}\right) U_{\lambda}^{-1}\right\} \\
& \quad=\operatorname{tr}\left(U(1-\lambda U)^{-1}-V_{\mu} U(1-\lambda U)^{-1} V_{\mu}^{-1}\right) .
\end{aligned}
$$

Obviously this does not depend on $\bar{\lambda}$, so that $\partial^{2} k(\lambda, \mu) / \partial \bar{\lambda} \partial \lambda=0$. By symmetry, $\partial^{2} k(\lambda, \mu) / \partial \bar{\mu} \partial \mu=0$, too.

\section{REFERENCES}

1. N. I. Akhiezer and I. M. Glazman, Theory of linear operators in Hilbert space, vols. I and II, Frederick Ungar Publ. Co., New York, 1962.

2. Kh. N. Boyadzhiev, Poisson integrals of the Pincus principal function for hyponormal operators, Rev. Roumaine Math. Pure Appl. 34 (1989), 197-211.

3. _ , , Trace formulas for commutators of unitary operators and for commutators of resolvents of unbounded self-adjoint operators, (Manuscript. Talk at the conference on Linear Operators and Applications-Oberwolfach, October 22-28, 1989.)

4. L. G. Brown, The determinant invariant for operators with trace class self-commutators, Lecture Notes in Math. vol. 345, 1973, pp. 210-228.

5. R. W. Carey and J. D. Pincus, Mosaics, principal functions, and mean motion in vonNeumann algebras, Acta Math. 138 (1977), 153-218.

6. _ , , Almost commuting pairs of unitary operators and flat currents, Integral Equations Operator Theory, 4 (1981), 45-122.

7. K. Clancey, Seminormal operators, Lecture Notes in Math., vol. 742, 1979.

8. __ A kernel for operators with one-dimensional self-commutator, Integral Equations Operator Theory, 7 (1984), 441-458.

9. _ Hilbert space operators with one-dimensional self-commutator, J. Operator Theory $\mathbf{1 3}$ (1985), 265-289.

10. J. Dixmier, Les algebres $d$ 'operateurs dans l'espace Hilbertien, Gauthier-Villars, Paris, 1969.

11. I. C. Gohberg and M. G. Krein, Introduction to the theory of linear non-self-adjoint Operators in Hilbert space, Trans. Math. Monographs 18 (1969).

12. J. W. Helton and R. Howe, Integral operators; commutator traces, index, and homology, Lecture Notes in Math., vol. 345, 1973, pp. 141-209.

13. P. Koosis, Introduction to $H_{p}$ spaces, London Math. Soc. Lecture Note Ser. 40 (1980).

14. M. Martin and M. Putinar, Lectures on hyponormal operators, Operator Theory: Adv. Appl. 39 (1989).

15. J. D. Pincus, On the trace of commutators in the algebra of operators generated by an operator with trace class self-commutator, 1972.

16. $\ldots$ Symmetric Wiener-Hopf and Toeplitz operators, Integral Equations Operator Theory 4 (1981), 123-133. 
17. J. D. Pincus, D. Xia, and J. Xia, An analytic model for operators with one-dimensional self-commutator, Integral Equations Operator Theory 7 (1984), 516-535.

18. E. M. Stein and G. Weiss, Introduction to Fourier analysis on Euclidean spaces, Princeton Univ. Press, Princeton, 1971.

19. D. Xia, Spectral theory of hyponormal operators, Operator Theory: Adv. Appl. 10 (1983).

20. __ On the analytic model of a class of hyponormal operators, Integral Equations Operator Theory 6 (1983), 134-157.

21. _ On the kernels associated with a class of hyponormal operators, Integral Equations Operator Theory 6 (1983), 444-452. 